\section{Long-term non- pharmacological weight loss interventions for adults with type 2 diabetes mellitus}

This is the abstract of a Cochrane Systematic Review published in the Cochrane Database of Systematic Reviews 2005, issue 2, art. no. CD004095. DOI: 10.1002/14651858.CD004095.pub2. For full text and details about the authors, see reference 1 .

\section{Susan L. Norris, Xuanping Zhang, Alison Avenell, Edward Gregg, Tamara Brown, Christopher H. Schmid, Joseph Lau}

The independent commentary was written by Adriano Namo Cury

\begin{abstract}
BACKGROUND: Most persons with type 2 diabetes are overweight and obesity worsens the metabolic and physiologic abnormalities associated with diabetes.

OBJECTIVE: The objective of this review is to assess the effectiveness of lifestyle and behavioral weight loss and weight control interventions for adults with type 2 diabetes.

METHODS:

Search methods: Studies were obtained from computerized searches of multiple electronic bibliographic databases, supplemented with hand searches of selected journals and consultation with experts in obesity research.

Selection criteria: Studies were included if they were published or unpublished randomized controlled trials in any language, and examined weight loss or weight control strategies using one or more dietary, physical activity, or behavioral interventions, with a follow-up interval of at least 12 months. Data collection and analysis: Effects were combined using a random effects model.

MAIN RESULTS: The 22 studies of weight loss interventions identified had a 4,659 participants and follow-up of 1 to 5 years. The pooled weight loss for any intervention in comparison to usual care among 585 subjects was $1.7 \mathrm{~kg}$ (95\% confidence interval [Cl] 0.3 to 3.2), or 3.1\% of baseline body weight among 517 subjects. Other main comparisons demonstrated non significant results: among 126 persons receiving a physical activity and behavioral intervention, those who also received a very low calorie diet lost $3.0 \mathrm{~kg}$ ( $95 \% \mathrm{Cl}-0.5$ to 6.4 ), or $1.6 \%$ of baseline body weight, more than persons receiving a low-calorie diet. Among 53 persons receiving identical dietary and behavioral interventions, those receiving more intense physical activity interventions lost $3.9 \mathrm{~kg}$ (95\% Cl -1.9 to 9.7), or 3.6\% of baseline body weight, more than those receiving a less intense or no physical activity intervention. Comparison groups often achieved significant weight loss (up to $10.0 \mathrm{~kg}$ ), minimizing between-group differences. Changes in glycated hemoglobin generally corresponded to changes in weight and were not significant when between-group differences were examined. No data were identified on quality of life and mortality.

AUTHORS CONCLUSIONS: Weight loss strategies using dietary, physical activity, or behavioral interventions produced small between-group improvements in weight. These results were minimized by weight loss in the comparison group, however, and examination of individual study arms revealed that multicomponent interventions including very low calorie diets or low calorie diets may hold promise for achieving weight loss in adults with type 2 diabetes.
\end{abstract}

The full text is available free-of-charge from: http://onlinelibrary.wiley. com/doi/10.1002/14651858.CD004095.pub2/abstract

The abstract is also available in the Portuguese and Chinese languages

\section{REFERENCE}

1. Norris SL, Zhang X, Avenell A, et al. Long-term non-pharmacologic weight loss interventions for adults with type 2 diabetes. Cochrane Database Syst Rev. 2005;(2):CD004095.

\section{COMMENTS}

The systematic review of 22 studies under the title "Long-term nonpharmacological weight loss interventions for adults with type 2 diabetes mellitus", by Norris et al., provides some reflections on how impactful the suggestions for the emphasis on optimization of lifestyle changes are, with regard to improving body weight, quality of life and biochemical markers among patients with type 2 diabetes.

Despite the heterogeneity of the studies involved, there are very few studies in the literature that have eschewed the pharmacological approach in favor of only targeting behavioral changes in habits, with and without physical activity, and thus focusing on weight loss, improvement of glycemic control and the impact on quality of life or even on mortality. Hence, this review will have an impact on this study subject.

The lack of statistical significance does not invalidate the changes in dietary behavior or lifestyle that should guide patients. This review demonstrates that weight loss does take place, but without great magnitude and sustainability beyond 12 months after the intervention. Very low-calorie and stricter diets present some advantages regarding weight loss, but the effect does not persist over long-term follow-up. No statistical strength was found, but there was always a trend towards improvement of weight or glycated hemoglobin levels for patients with type 2 diabetes who underwent intervention and monitoring.

The number of homogeneous studies is certainly insufficient to establish waymarks and rules. Good sense needs to prevail in dietary counseling and physical activity. Even if this non-pharmacological intervention is not shown to have any significant impact on mortality, the set of therapies proposed for patients with type 2 diabetes will certainly have an impact and will give rise to improvement of quality of life and understanding of the disease, even if the weight loss effect becomes diluted with time. There is an ever-present need to seek strategies to control chronic diseases, so as to achieve health benefits that transform the statistics.

It is noteworthy that a recent study in the New England Journal of Medicine $^{2}$ showed that the type of diet can indeed make a difference in this type of study. Different paths need to be devised for different patients, so that the full meaning of treatment can be attained.

Adriano Namo Cury, MD, PhD. Professor, School of Medical Sciences, Santa Casa de São Paulo (FCMSCSP), São Paulo, Brazil.

\section{REFERENCES}

1. Norris SL, Zhang X, Avenell A, et al. Long-term non-pharmacologic weight loss interventions for adults with type 2 diabetes. Cochrane Database Syst Rev. 2005;(2):CD004095.

2. Estruch R, Ros E, Salas-Salvadó J, et al. Primary prevention of cardiovascular disease with a Mediterranean diet. N Engl J Med. 2013;368(14):1279-90. 